ESAIM: PROCEEDINGS, October 2007, Vol.20, 16-28

Mohammed-Najib Benbourhim, Patrick Chenin, Abdelhak Hassouni \& Jean-Baptiste Hiriart-Urruty, Editors

\title{
SUBDIVISION SCHEME OF QUARTIC BIVARIATE SPLINES ON A FOUR-DIRECTIONAL MESH
}

\author{
El Bachir Ameur ${ }^{1}$, Domingo Barrera Rosillo ${ }^{2}$ and Driss Sbibih $^{3}$
}

\begin{abstract}
In this paper we give a new definition of minimally and quasi-minimally supported $\mathcal{C}^{2}$ quartic bivariate B-splines associated with the four-directional mesh of the plane, introduced in $[7,19]$, which is convenient to show that theses B-splines satisfy the refinement equation and we determine the associated matrix mask, we prove that the family of these B-splines is stable and the associated subdivision scheme converges. These results can be extended to various cases in the spline space of class $\mathcal{C}^{3 k+2}$ and degree $4 k+4$, but in these cases the supports of the masks are larger.
\end{abstract}

Résumé. Dans cet article nous donnons une nouvelle définition des B-splines quartiques de classe $\mathcal{C}^{2}$ à support minimal et quasi-minimal sur le réseau quadridirectionnel du plan, introduites dans $[7,19]$. Nous utilisons cette définition pour montrer que ces B-splines vérifient une équation de raffinement et déterminer les matrices du filtre associé. Nous montrons que la famille de ces B-splines est stable et que le schéma de subdivision associé converge. Ces résultats peuvent être généralisés aux espaces de fonctions splines de classe $\mathcal{C}^{3 k+2}$ et de degré $4 k+4$, mais dans ce cas les supports des filtres associés deviennent plus grands.

\section{INTRODUCTION}

Refinable function vectors and vector subdivision schemes, as two of the most important and extensively studied fundamental objects in the literature of wavelet analysis, are useful in many applications such as signal processing and computer aided geometric design ( $[4,5,9,10,13,16,22])$. In $[9,10]$ the authors give the subdivision scheme associated with the function vectors of two linear and cubic B-splines. In this paper we are interested in the function vector of three $\mathcal{C}^{2}$ quartic B-splines on the four-directional mesh.

We begin with some notations and definitions used throughout this paper. Let $\tau$ be the uniform triangulation of the plane, whose set of vertices is $\mathbb{Z}^{2}$, and whose edges are parallel to the four directions $e_{1}=(1,0), e_{2}=(0,1)$, $e_{3}=(1,1)$ and $e_{4}=(1,-1)$. This type of triangulation is called a four-directional mesh. For any integers $r$ and $d$, let $\mathbb{P}_{d}$ be the space of bivariate polynomials of total degree at most $d$, and

$$
\mathcal{S}_{d}^{r}(\tau):=\left\{s \in \mathcal{C}^{r}\left(\mathbb{R}^{2}\right):\left.s\right|_{T} \in \mathbb{P}_{d} \text { for all } T \in \tau\right\}
$$

\footnotetext{
1 Université Moulay Ismail, Faculté des sciences et Techniques, Département d'Informatique, 52000 Errachdia, Maroc;email: ameurelbachir@yahoo.fr

2 Departamento de Matemática Aplicada, Faculdad de Ciencias, Universidad de Granada, Campus Universitario de Fuentenueva s/n, 18071, Granada, Spain; e-mail: dbarrera@ugr.es

3 Université Mohammed I, Ecole Suprieure de Technologie, Laboratoire MATSI, Oujda, Maroc; e-mail: sbibih@yahoo.fr
}

(C) EDP Sciences, SMAI 2007 
be the space of bivariate piecewise polynomial functions of class $\mathcal{C}^{r}$ on the plane and whose restrictions to each triangular cell of $\tau$ are in $\mathbb{P}_{d}$.

Let $T$ be a triangle of $\tau$ and $\lambda=\left(\lambda_{1}, \lambda_{2}, \lambda_{3}\right)$ be the barycentric coordinates of a point $M$ of $\mathbb{R}^{2}$ relative to $T$. Each polynomial $p$ of $\mathbb{P}_{d}(T)$ has a unique representation in the Bernstein-Bézier form:

$$
p(M)=\sum_{\mu \in \triangle_{d}} b(\mu) B_{\mu}^{d}(\lambda)
$$

where

and

$$
\triangle_{d}=\left\{\mu=\left(\mu_{1}, \mu_{2}, \mu_{3}\right) \in \mathbb{Z}_{+}^{3}:|\mu|=\mu_{1}+\mu_{2}+\mu_{3}=d\right\}
$$

$$
B_{\mu}^{d}(\lambda)=\frac{d !}{\mu !} \lambda^{\mu}=\frac{d !}{\mu_{1} ! \mu_{2} ! \mu_{3} !} \lambda_{1}^{\mu_{1}} \lambda_{2}^{\mu_{2}} \lambda_{3}^{\mu_{3}} .
$$

The family of the $\left(\begin{array}{c}d+2 \\ 2\end{array}\right)$ polynomials $B_{\mu}^{d}, \mu \in \triangle_{d}$, forms a basis for the space $\mathbb{P}_{d}(T)$. The coefficients $\left\{b(\mu), \mu \in \triangle_{d}\right\}$, are called the B-net of $p$ on the triangle $T$.

When $r=0$ and $d=1$, the linear bivariate splines space on the four-directional mesh $\tau, \mathcal{S}_{1}^{0}(\tau)$, is generated by two minimally supported linear B-splines $\varphi_{1}$ and $\varphi_{2}$, whose B-nets and supports are given in Figure 1.
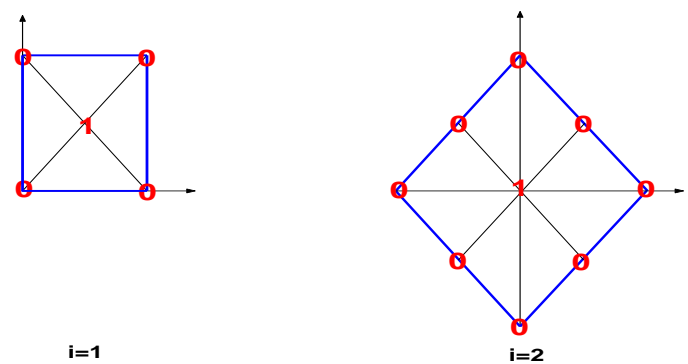

Figure 1. B-nets and supports of $\varphi_{i}$ for $i=1,2$.

A bivariate compactly supported function vector $\Psi=\left(\psi_{1}, \cdots, \psi_{m}\right)^{T}$ is said to be refinable if it satisfies the refinement equation

$$
\Psi=\sum_{j \in \mathbb{Z}^{2}} P_{j} \Psi(2 \cdot-j)
$$

where the sequence $\mathbf{P}:=\left(P_{j}\right)_{j \in \mathbb{Z}^{2}}$ is the refinement matrix mask with $P_{j}$ being real $(m \times m)$-matrices.

Let $\left(\ell\left(\mathbb{Z}^{2}\right)\right)$ and $\left(\ell^{\infty}\left(\mathbb{Z}^{2}\right)\right)$ be the linear space of all sequences on $\mathbb{Z}^{2}$ and the subspace of all bounded sequences respectively. We say that $\Psi$ is stable if its integer translates satisfy the stability condition, i.e., if there exist constants $0<c_{1} \leq c_{2}<\infty$ such that

$$
c_{1}\|\lambda\|_{\infty} \leq\left\|\sum_{j \in \mathbb{Z}^{2}} \lambda_{j}^{T} \Psi(\cdot-j)\right\|_{\infty} \leq c_{2}\|\lambda\|_{\infty} \text { far all } \lambda \in\left(\ell^{\infty}\left(\mathbb{Z}^{2}\right)\right)^{m} .
$$

where $\|\lambda\|_{\infty}=\max _{i}\left\|\lambda^{i}\right\|_{\infty}$ and $\|f\|_{\infty}$ denote the sup-norms of the vector sequence $\lambda$ and the bivariate function $f$ respectively.

The subdivision operator associated with $\mathbf{P}$ is given by

$$
\begin{aligned}
& \mathbb{S}_{P}:\left(\ell\left(\mathbb{Z}^{2}\right)\right)^{m} \longrightarrow\left(\ell\left(\mathbb{Z}^{2}\right)\right)^{m} \\
& \left(\mathbb{S}_{P} \lambda\right)_{i}:=\sum_{j \in \mathbb{Z}^{2}} P_{i-2 j}^{T} \lambda_{j}, \quad i \in \mathbb{Z}^{2},
\end{aligned}
$$


In this paper we are interested in the quartic bivariate splines space $\mathcal{S}_{4}^{2}(\tau)$ on the four-directional mesh $\tau$, which is generated by three independent locally supported B-splines, two are minimally supported $\psi_{1}$ and $\psi_{2}$, they are constructed by Sablonnière in [18], whose B-nets and supports are given in the Figure 2, and one is quasi-minimally supported $\psi_{3}$ constructed by Chui and He in [7], whose B-net and support are given in Figure 3 .
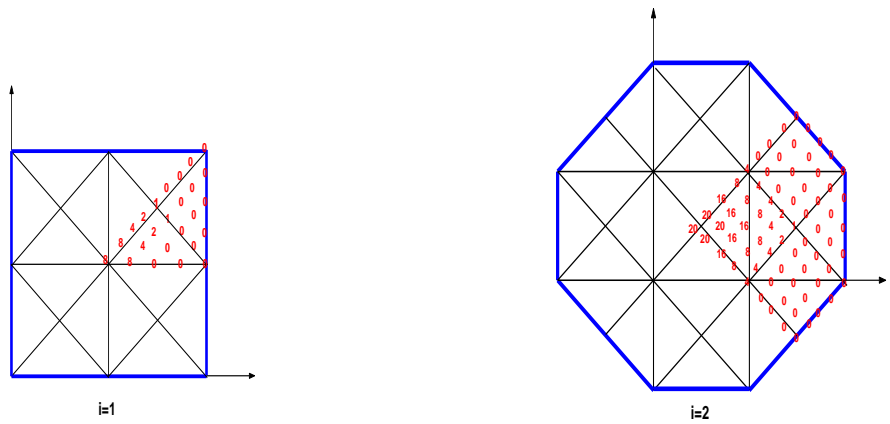

Figure 2. B-nets and supports of $48 \psi_{i}$ for $i=1,2$.

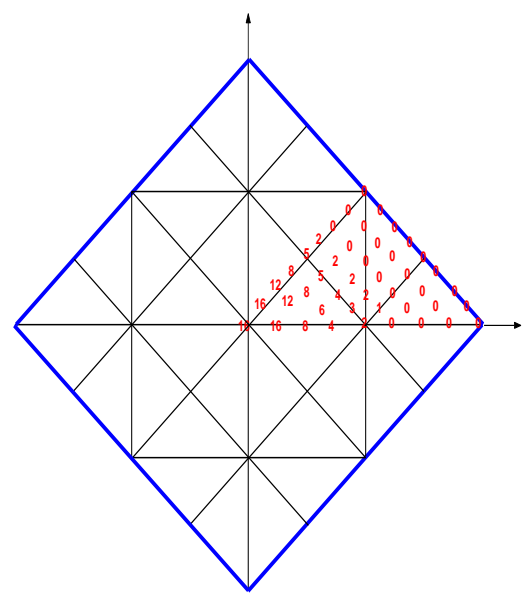

Figure 3. B-net and support of $48 \psi_{3}$.

Using only the B-nets of these B-splines, it is difficult to show that neither these B-splines satisfy a refinement equation nor determine their associated matrix masks. So that, in this section 1, we give a new definition of these B-splines which is convenient to prove that the function vector $\left(\psi_{1}, \psi_{2}, \psi_{3}\right)^{T}$ satisfies the refinement equation and we can determine explicitly the associated refinement matrix mask. Moreover, we prove that this function vector is stable and the associated subdivision scheme converges, which is the objective of the section 2 . 


\section{Definition of quartic B-Splines $\psi_{1}, \psi_{2}, \psi_{3}$ BY Convolution}

Let $\Psi:=\left(\psi_{1}, \psi_{2}, \psi_{3}\right)^{T}$ be the function vector of the above three quartic B-splines in the space $\mathcal{S}_{4}^{2}(\tau)$. In this section we give a new definition of these B-splines which is convenient to prove that the function vector $\Psi$ verifies the refinement equation:

$$
\Psi=\sum_{j \in \mathbb{Z}^{2}} P_{j} \Psi(2 \cdot-j)
$$

and we can determine explicitly the associated refinement matrix mask $\mathbf{P}:=\left(P_{j}\right)_{j \in \mathbb{Z}^{2}}$ where $P_{j}$ is a real $3 \times 3$-matrix, for all $j \in \mathbb{Z}^{2}$.

Lemma 1.1. The B-splines $\varphi_{1} * \varphi_{1}, \varphi_{1} * \varphi_{2}$ and $\varphi_{2} * \varphi_{2}$ satisfy the following symmetry properties:

$$
\begin{gathered}
\varphi_{1} * \varphi_{1}(\cdot)=\varphi_{1} * \varphi_{1}\left(G_{i} \cdot+2 \eta_{i}\right), \\
\varphi_{1} * \varphi_{2}(\cdot)=\varphi_{1} * \varphi_{2}\left(G_{i} \cdot+\eta_{i}\right)
\end{gathered}
$$

and

for $i=0,1$, where

$$
\varphi_{2} * \varphi_{2}(\cdot)=\varphi_{2} * \varphi_{2}\left(G_{i} \cdot\right)
$$

$$
G_{0}=\left(\begin{array}{ll}
0 & 1 \\
1 & 0
\end{array}\right), G_{1}=-G_{0}, \eta_{0}=(0,0), \eta_{1}=(1,1)
$$

and $*$ denote the convolution operator of the scaling functions.

Proof. Using the fact that the linear B-splines $\varphi_{1}$ and $\varphi_{2}$ satisfy the symmetry properties

$$
\varphi_{1}(\cdot)=\varphi_{1}\left(\cdot G_{i}+\eta_{i}\right) \text { and } \varphi_{2}(\cdot)=\varphi_{2}\left(\cdot G_{i}\right)
$$

we deduce that, for $i=0,1$,

$$
\begin{aligned}
\varphi_{1} * \varphi_{1}(x, y) & =\int_{\mathbb{R}^{2}} \varphi_{1}(u, v) \varphi_{1}(x-u, y-v) d u d v \\
& =\int_{\operatorname{supp} \varphi_{1}} \varphi_{1}\left((u, v) G_{i}+\eta_{i}\right) \varphi_{1}\left((x-u, y-v) G_{i}+\eta_{i}\right) d u d v .
\end{aligned}
$$

If we put $(s, t)=(u, v) G_{i}+\eta_{i}$, then, by using a variable change, we obtain

$$
\varphi_{1} * \varphi_{1}(x, y)=\left|\operatorname{det} G_{i}\right|^{2} \int_{\operatorname{supp} \varphi_{1} G_{i}+\eta_{i}} \varphi_{1}(s, t) \varphi_{1}\left((x, y) G_{i}+2 \eta_{i}-(s, t)\right) d s d t
$$

since $\left(\operatorname{supp} \varphi_{1}\right) G_{i}+\eta_{i}=\operatorname{supp} \varphi_{1}$ and $\left|\operatorname{det} G_{i}\right|=1$, we obtain

$$
\varphi_{1} * \varphi_{1}(x, y)=\int_{\operatorname{supp} \varphi_{1}} \varphi_{1}(s, t) \varphi_{1}\left((x, y) G_{i}+2 \eta_{i}-(s, t)\right) d s d t
$$

consequently, we have

$$
\varphi_{1} * \varphi_{1}(x, y)=\varphi_{1} * \varphi_{1}\left((x, y) G_{i}+2 \eta_{i}\right)
$$

A similar technique can be applied to obtain the equations (6) and (7).

Theorem 1.2. The minimally and quasi-minimally supported quartic B-splines $\psi_{1}, \psi_{2}$ and $\psi_{3}$ are defined by:

$$
\Psi:=\left[\begin{array}{c}
\psi_{1} \\
\psi_{2} \\
\psi_{3}
\end{array}\right]=\left[\begin{array}{c}
\varphi_{1} * \varphi_{1} \\
2 \varphi_{1} * \varphi_{2} \\
\varphi_{2} * \varphi_{2}
\end{array}\right]
$$


Proof. Firstly, it is easy to verify that the supports of the B-splines $\varphi_{1} * \varphi_{1}, \varphi_{1} * \varphi_{2}$ and $\varphi_{2} * \varphi_{2}$ are the same of those of $\psi_{1}, \psi_{2}$ and $\psi_{3}$ respectively illustrated in figures 2 and 3 .

Hence, to prove this theorem, it suffices to compute the B-nets of these B-splines on their supports. From Lemma 1.1, the symmetry properties of the B-splines $\varphi_{1} * \varphi_{1}, \varphi_{1} * \varphi_{2}$ and $\varphi_{2} * \varphi_{2}$ allow us to compute their B-nets only on a part of their supports. For this, since the linear box spline $B_{1,1,1,0}$ lies in $\mathcal{S}_{1}^{0}(\tau)($ see $[6])$, we can easily verify that

consequently

$$
B_{1,1,1,0}=\frac{1}{2} \varphi_{1}+\frac{1}{2} \varphi_{1}\left(\cdot-e_{3}\right)+\varphi_{2}\left(\cdot-e_{3}\right)
$$

and

$$
\varphi_{1} * B_{1,1,1,0}=\frac{1}{2} \varphi_{1} * \varphi_{1}+\frac{1}{2} \varphi_{1} * \varphi_{1}\left(\cdot-e_{3}\right)+\varphi_{1} * \varphi_{2}\left(\cdot-e_{3}\right),
$$

$$
\varphi_{2} * B_{1,1,1,0}=\frac{1}{2} \varphi_{1} * \varphi_{2}+\frac{1}{2} \varphi_{1} * \varphi_{2}\left(\cdot-e_{3}\right)+\varphi_{2} * \varphi_{2}\left(\cdot-e_{3}\right) .
$$

However, we can compute easily the B-nets of the B-splines $\varphi_{1} * B_{1,1,1,0}$ and $\varphi_{2} * B_{1,1,1,0}$ by using the classical algorithm for generating the B-nets of bivariate B-splines defined on the four-direction mesh developed in [8].

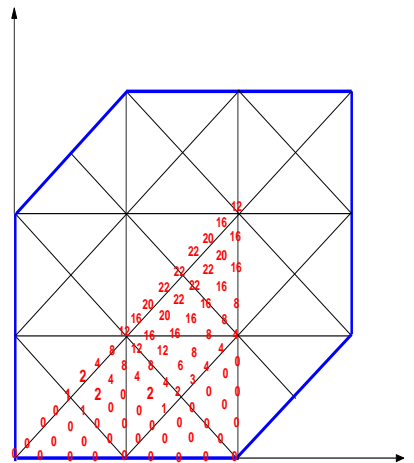

$\mathrm{i}=1$

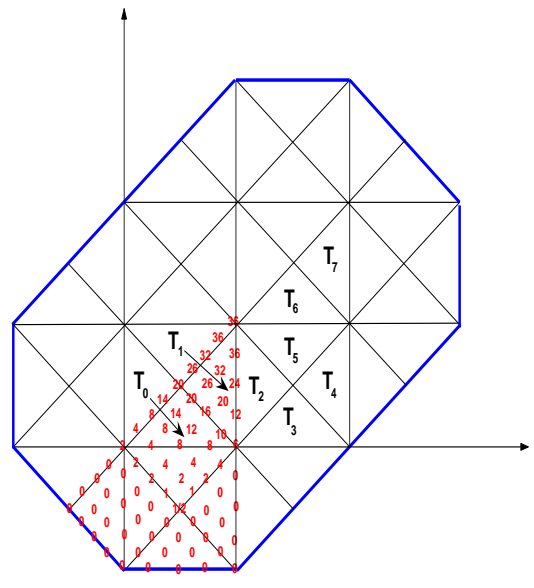

$\mathrm{i}=2$

Figure 4. B-nets and supports of $96 \varphi_{i} * B_{1,1,1,0}, i=1,2$, and the triangles $T_{i}$ for $i=0, \cdots, 7$

Now, by using the equations (9) and (10) we obtain

$$
\begin{gathered}
\left.\left(\varphi_{1} * \varphi_{1}\right)\right|_{T_{0}}=\left.2\left(\varphi_{1} * B_{1,1,1,0}\right)\right|_{T_{0}}, \\
\left.\left(\varphi_{1} * \varphi_{1}\right)\right|_{T_{1}}=\left.2\left(\varphi_{1} * B_{1,1,1,0}\right)\right|_{T_{1}}-\left.4\left(\varphi_{2} * B_{1,1,1,0}\left(\cdot-e_{3}\right)\right)\right|_{T_{1}}, \\
\left.\left(\varphi_{1} * \varphi_{2}\left(\cdot-e_{3}\right)\right)\right|_{T_{1} \cup T_{2} \cup T_{3}}=\left.2\left(\varphi_{1} * B_{1,1,1,0}\right)\right|_{T_{1}} \cup T_{2} \cup T_{3}, \\
\left.\left(\varphi_{1} * \varphi_{2}\left(\cdot-e_{3}\right)\right)\right|_{T_{4} \cup T_{5}}=\left.\left(\varphi_{1} * B_{1,1,1,0}\right)\right|_{T_{4} \cup T_{5}}-\left.\frac{1}{2}\left(\varphi_{1} * \varphi_{1}\right)\right|_{T_{4} \cup T_{5}}, \\
\left.\left(\varphi_{1} * \varphi_{2}\left(\cdot-e_{3}\right)\right)\right|_{T_{6}}=\left.\left(\varphi_{1} * B_{1,1,1,0}\right)\right|_{T_{6}}-\left.\frac{1}{2}\left(\varphi_{1} * \varphi_{1}\right)\right|_{T_{6}}-\left.\frac{1}{2}\left(\varphi_{1} * \varphi_{1}\left(\cdot-e_{3}\right)\right)\right|_{T_{6}}, \\
\left.\left(\varphi_{2} * \varphi_{2}\left(\cdot-2 e_{3}\right)\right)\right|_{T_{4} \cup T_{5} \cup T_{6}}=\left.\left(\varphi_{2} * B_{1,1,1,0}\left(\cdot-e_{3}\right)\right)\right|_{T_{4} \cup T_{5} \cup T_{6}}-\left.\frac{1}{2}\left(\varphi_{1} * \varphi_{2}\left(\cdot-2 e_{3}\right)\right)\right|_{T_{4} \cup T_{5} \cup T_{6}}
\end{gathered}
$$




$$
\left.\left(\varphi_{2} * \varphi_{2}\left(\cdot-2 e_{3}\right)\right)\right|_{T_{7}}=\left.\left(\varphi_{2} * B_{1,1,1,0}\left(\cdot-e_{3}\right)\right)\right|_{T_{7}}-\left.\frac{1}{2}\left(\varphi_{1} * \varphi_{2}\left(\cdot-e_{3}\right)\right)\right|_{T_{7}}-\left.\frac{1}{2}\left(\varphi_{1} * \varphi_{2}\left(\cdot-2 e_{3}\right)\right)\right|_{T_{7}} .
$$

where the triangles $T_{i}, i=0, \cdots, 7$ are illustrated in Figure 4.

In the first step, we compute the B-net of the B-spline $\varphi_{1} * \varphi_{1}$ by using the equations (11) and (12), and the symmetry properties of this B-spline (5). In the second step, we use the equations (6), (13), (14) and (15), and the B-net of $\varphi_{1} * \varphi_{1}$ for computing that of $\varphi_{1} * \varphi_{2}$. Finally, by using the the equations (7), (16) and (17), and the B-net of $\varphi_{1} * \varphi_{2}$, we compute that of $\varphi_{2} * \varphi_{2}$. After the computation, we obtain the equation (8).

\section{REFINEMENT EQUATION AND SUBDIVISION SCHEME}

In this section, we determine the refinement equation associated with the function vector $\Psi$ of the three quartic B-splines $\psi_{1}, \psi_{2}$ and $\psi_{3}$ by using the new definition described above and the following lemmas (see [9]). Furthermore, we prove that this refinable function vector $\Psi$ is stable and the associated subdivision scheme converges.

Lemma 2.1. Let $\Lambda=\left(\lambda_{1}, \cdots, \lambda_{n}\right)^{T}$ and $\Theta=\left(\theta_{1}, \cdots, \theta_{m}\right)^{T}$ be two refinable function vectors of compactly supported bivariate functions, satisfying the refinement equations

$$
\Lambda=\sum_{j \in \mathbb{Z}^{2}} A_{j} \Lambda(2 \cdot-j)
$$

and

$$
\Theta=\sum_{j \in \mathbb{Z}^{2}} B_{j} \Theta(2 \cdot-j)
$$

with corresponding matrix masks $\mathbf{A}:=\left(A_{j}\right)_{j \in \mathbb{Z}^{2}}$ and $\mathbf{B}:=\left(B_{j}\right)_{j \in \mathbb{Z}^{2}}$, which are matrix sequences of $(n \times n)$ matrices and $(m \times m)$-matrices respectively. Then the Kronecker convolved function vector

$$
\Lambda * \Theta:=\left(\begin{array}{c}
\lambda_{1} * \Theta \\
\vdots \\
\lambda_{n} * \Theta
\end{array}\right):=\left(\begin{array}{c}
\lambda_{1} * \theta_{1} \\
\vdots \\
\lambda_{1} * \theta_{m} \\
\vdots \\
\lambda_{n} * \theta_{1} \\
\vdots \\
\lambda_{n} * \theta_{m}
\end{array}\right)
$$

verifies the following refinement equation

$$
\Lambda * \Theta=\sum_{j \in \mathbb{Z}^{2}} C_{j} \Lambda * \Theta(2 \cdot-j)
$$

where the refinement mask $\mathbf{C}:=\left(C_{j}\right)_{j \in \mathbb{Z}^{2}}$, which is a matrix sequence of $(n m \times n m)$-matrices, is computed as follows:

$$
C_{j}=\frac{1}{4} \sum_{i \in \mathbb{Z}^{2}} A_{i} \otimes B_{j-i}, \quad j \in \mathbb{Z}^{2}
$$

with $\otimes$ denotes the Kronecker product of matrices.

Lemma 2.2. The function vector $\Phi:=\left(\varphi_{1}, \varphi_{2}\right)^{T}$ of linear bivariate B-splines on a four-direction mesh satisfies the matrix refinement equation

$$
\Phi=\sum_{j \in \mathbb{Z}^{2}} H_{j} \Phi(2 \cdot-j)
$$


where the refinement matrix mask $\mathbf{H}:=\left(H_{j}\right)_{j \in \mathbb{Z}^{2}}$, which is a matrix sequence of $(2 \times 2)$-matrices, is given by

$$
\begin{aligned}
& j_{1}=0
\end{aligned}
$$

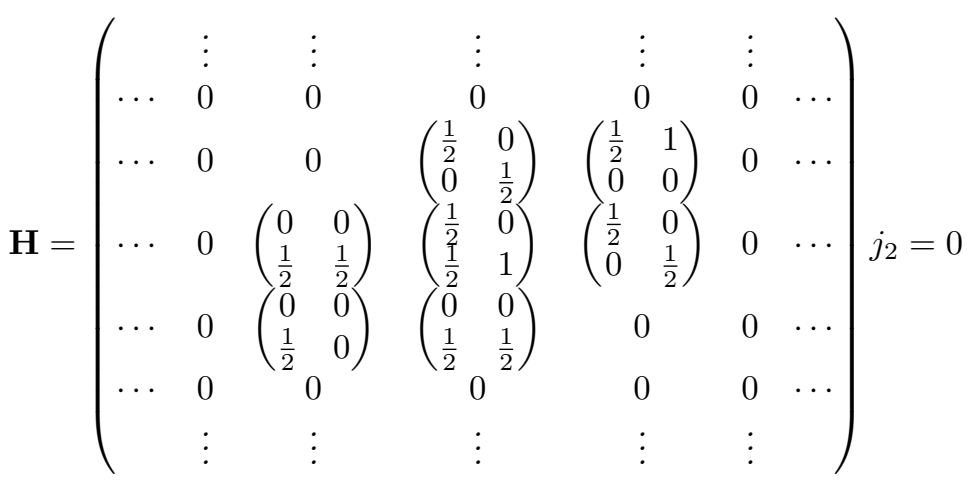

Theorem 2.3. The function vector $\Psi:=\left(\psi_{1}, \psi_{2}, \psi_{3}\right)^{T}$ of a $\mathcal{C}^{2}$ quartic minimally and quasi-minimally supported bivariate B-spline on a four-direction mesh satisfies the following refinement equation:

$$
\Psi=\sum_{j \in \mathbb{Z}^{2}} P_{j} \Psi(2 \cdot-j)
$$

where the refinement matrix mask $\mathbf{P}:=\left(P_{j}\right)_{j \in \mathbb{Z}^{2}}$, which is a matrix sequence of $(3 \times 3)$-matrices, is given by

$$
\begin{gathered}
P_{(0,0)}=\left[\begin{array}{ccc}
\frac{1}{16} & 0 & 0 \\
\frac{1}{2} & \frac{3}{8} & 0 \\
\frac{1}{16} & \frac{1}{4} & \frac{1}{2}
\end{array}\right], P_{(1,0)}=\left[\begin{array}{ccc}
\frac{1}{8} & 0 & 0 \\
\frac{1}{4} & \frac{3}{8} & \frac{1}{4} \\
0 & \frac{1}{16} & \frac{1}{4}
\end{array}\right], P_{(2,0)}=\left[\begin{array}{ccc}
\frac{1}{16} & 0 & 0 \\
0 & \frac{1}{16} & 0 \\
0 & 0 & \frac{1}{16}
\end{array}\right], \\
P_{(1,1)}=\left[\begin{array}{lll}
\frac{1}{4} & \frac{1}{8} & 0 \\
\frac{1}{8} & \frac{3}{8} & \frac{1}{2} \\
0 & 0 & \frac{1}{8}
\end{array}\right], P_{(2,1)}=\left[\begin{array}{ccc}
\frac{1}{8} & \frac{1}{8} & 0 \\
0 & \frac{1}{16} & \frac{1}{4} \\
0 & 0 & 0
\end{array}\right], P_{(2,2)}=\left[\begin{array}{ccc}
\frac{1}{16} & \frac{1}{8} & \frac{1}{4} \\
0 & 0 & 0 \\
0 & 0 & 0
\end{array}\right], \\
P_{(-1,-1)}=\left[\begin{array}{lll}
0 & 0 & 0 \\
\frac{1}{8} & 0 & 0 \\
\frac{1}{4} & \frac{1}{4} & \frac{1}{8}
\end{array}\right], P_{(0,-1)}=\left[\begin{array}{ccc}
0 & 0 & 0 \\
\frac{1}{4} & \frac{1}{16} & 0 \\
\frac{1}{8} & \frac{1}{4} & \frac{1}{4}
\end{array}\right], P_{(1,-1)}=\left[\begin{array}{ccc}
0 & 0 & 0 \\
\frac{1}{8} & \frac{1}{16} & 0 \\
0 & \frac{1}{16} & \frac{1}{8}
\end{array}\right], \\
P_{(0,-2)}=\left[\begin{array}{ccc}
0 & 0 & 0 \\
0 & 0 & 0 \\
\frac{1}{16} & \frac{1}{16} & \frac{1}{16}
\end{array}\right], P_{(-1,-2)}=\left[\begin{array}{ccc}
0 & 0 & 0 \\
0 & 0 & 0 \\
\frac{1}{8} & \frac{1}{16} & 0
\end{array}\right], P_{(-2,-2)}=\left[\begin{array}{ccc}
0 & 0 & 0 \\
0 & 0 & 0 \\
\frac{1}{16} & 0 & 0
\end{array}\right], \\
P_{j}=P_{G_{0} j} \text { for all } j \in\{(-2,-1),(-2,0),(-1,0),(-1,1),(0,1),(0,2),(1,2)\},
\end{gathered}
$$

and

$$
P_{j}=0 \text { for all } j \in \mathbb{Z}^{2} \backslash \mathcal{H}_{2}
$$

where $\mathcal{H}_{2}$ is the subset of $\mathbb{Z}^{2}$ which intersect the hexagon of vertices $\{(-2,-2),(0,-2),(2,0),(2,2),(0,2),(-2,0)\}$.

Proof. From lemmas 2.1 and 2.2, it follows that the Kronecker convolved function vector $\Phi * \Phi$ satisfies the following refinement equation

$$
\Phi * \Phi=\sum_{j \in \mathbb{Z}^{2}} \widetilde{H}_{j} \Phi * \Phi(2 \cdot-j)
$$


where the matrix sequence of $(4 \times 4)$-matrices $\left(\widetilde{H}_{j}\right)_{j \in \mathbb{Z}^{2}}$ is given by

$$
\widetilde{H}_{j}=\frac{1}{4} \sum_{i \in \mathbb{Z}^{2}} H_{i} \otimes H_{j-i}, \quad j \in \mathbb{Z}^{2}
$$

On the other hand

$$
\Psi=R \Phi * \Phi
$$

with

and

$$
R=\left[\begin{array}{llll}
1 & 0 & 0 & 0 \\
0 & 1 & 1 & 0 \\
0 & 0 & 0 & 1
\end{array}\right]
$$

with

$$
\Phi * \Phi=T \Psi
$$

$$
T=\left[\begin{array}{lll}
1 & 0 & 0 \\
0 & \frac{1}{2} & 0 \\
0 & \frac{1}{2} & 0 \\
0 & 0 & 1
\end{array}\right]
$$

Hence, by using the equations (24) and (30) we obtain

$$
\Psi=R \Phi * \Phi=\sum_{j \in \mathbb{Z}^{2}} R \widetilde{H}_{j} \Phi * \Phi(2 \cdot-j)=\sum_{j \in \mathbb{Z}^{2}} R \widetilde{H}_{j} T \Psi(2 \cdot-j)
$$

consequently we have

$$
P_{j}=R \widetilde{H}_{j} T=\frac{1}{4} \sum_{i \in \mathbb{Z}^{2}} R\left(H_{i} \otimes H_{j-i}\right) T, \quad j \in \mathbb{Z}^{2} .
$$

Then, by using the matrix mask $H_{j}$ described in Lemma 2.2, we determine explicitly the matrix mask $P_{j}$.

Now, we use the refinement equation (23) to define the subdivision scheme associated with the refinable function vector $\Psi$ as follows.

$$
\begin{aligned}
& \text { For a given fuction spline } S_{\lambda}=\sum_{j \in \mathbb{Z}^{2}} \lambda_{j}^{T} \Psi(\cdot-j) \text {. } \\
& \text { Put } \lambda^{(0)}=\lambda \text { and } \\
& \text { Compute } \lambda_{i}^{(n)}=\sum_{j \in \mathbb{Z}^{2}} P_{i-2 j}^{T} \lambda_{j}^{(n-1)}, \quad i \in \mathbb{Z}^{2}, n=1,2, \cdots
\end{aligned}
$$

Following [10], we say that the subdivision scheme converges for $\lambda=\left(\lambda^{1}, \lambda^{2}, \lambda^{3}\right)^{T} \in\left(\ell^{\infty}\left(\mathbb{Z}^{2}\right)\right)^{3}$ if there exists a continuous function $S_{\lambda}: \mathbb{R}^{2} \longrightarrow \mathbb{R}$ such that

$$
\lim _{n \rightarrow \infty}\left\|S_{\lambda}\left(\frac{\cdot}{2^{n}}\right) \mathbf{e}-\lambda^{(n)}\right\|_{\infty} \text { for } \mathbf{e}=(1,1,1)^{T}
$$

The symbol $S_{\lambda}\left(\frac{\cdot}{2^{n}}\right)$ stands for the scalar-valued sequence $\left(S_{\lambda}\left(\frac{j}{2^{n}}\right)\right)_{j \in \mathbb{Z}^{2}}$.

Lemma 2.4. Let $\Theta=\left(\theta_{1}, \cdots, \theta_{m}\right)^{T}$ be a refinable function vector of continuous compactly supported bivariate functions, satisfying the refinement equation

$$
\Theta=\sum_{j \in \mathbb{Z}^{2}} B_{j} \Theta(2 \cdot-j)
$$


If $\Theta$ is stable and the associated matrix mask $\mathbf{B}:=\left(B_{j}\right)_{j \in \mathbb{Z}^{2}}$ satisfies

$$
\mathbf{v} \sum_{j \in \mathbb{Z}^{2}} B_{i-2 j}=\mathbf{v}, \quad i \in \mathbb{Z}^{2}, \text { for } \mathbf{v}=(1,1, \cdots, 1),
$$

then the associated subdivision scheme is convergent for all $\lambda \in\left(\ell^{\infty}\left(\mathbb{Z}^{2}\right)^{m}\right.$, and the limit function is given by

$$
S_{\lambda}=\sum_{j \in \mathbb{Z}^{2}} \lambda_{j}^{T} \Theta(\cdot-j)
$$

Lemma 2.5. The $\mathcal{C}^{2}$ quartic spline function vector $\Psi$ is stable.

Proof. From [16], $\Psi$ is stable if and only if the sequences

$$
\left(\widehat{\psi}_{r}(\omega+2 \pi j)\right)_{j \in \mathbb{Z}^{2}} \quad r=1,2,3
$$

are linearly independent for every $\omega \in \mathbb{R}^{2}$. Suppose that $\Psi$ is not stable, then there exist $\omega_{0} \in \mathbb{R}^{2}, j_{0} \in \mathbb{Z}^{2}$ and $r_{0} \in\{1,2,3\}$ such that

$$
S\left(\omega_{0}\right)=\sum_{r=1}^{3} \sum_{j \in \mathbb{Z}^{2}} c_{j}^{r} \widehat{\psi}_{r}\left(\omega_{0}+2 \pi j\right)=0 \text { with } c_{j_{0}}^{r_{0}} \neq 0 .
$$

By using the new definition of $\Psi$ described in Theorem 1.2, we obtain

$$
\begin{aligned}
S\left(\omega_{0}\right)= & \sum_{j \in \mathbb{Z}^{2}}\left\{c_{j}^{1} \widehat{\varphi}_{1}\left(\omega_{0}+2 \pi j\right) \widehat{\varphi}_{1}\left(\omega_{0}+2 \pi j\right)+2 c_{j}^{2} \widehat{\varphi}_{1}\left(\omega_{0}+2 \pi j\right) \widehat{\varphi}_{2}\left(\omega_{0}+2 \pi j\right)\right. \\
& \left.+\quad c_{j}^{3} \widehat{\varphi}_{2}\left(\omega_{0}+2 \pi j\right) \widehat{\varphi}_{2}\left(\omega_{0}+2 \pi j\right)\right\} \\
= & \sum_{j \in \mathbb{Z}^{2}}\left\{c_{j}^{1} \widehat{\varphi}_{1}\left(\omega_{0}+2 \pi j\right)+c_{j}^{2} \widehat{\varphi}_{2}\left(\omega_{0}+2 \pi j\right)\right\} \widehat{\varphi}_{1}\left(\omega_{0}+2 \pi j\right) \\
& +\quad\left\{c_{j}^{2} \widehat{\varphi}_{1}\left(\omega_{0}+2 \pi j\right)+c_{j}^{3} \widehat{\varphi}_{2}\left(\omega_{0}+2 \pi j\right)\right\} \widehat{\varphi}_{2}\left(\omega_{0}+2 \pi j\right) .
\end{aligned}
$$

If we put

and

$$
d_{j}^{1}=c_{j}^{1} \widehat{\varphi}_{1}\left(\omega_{0}+2 \pi j\right)+c_{j}^{2} \widehat{\varphi}_{2}\left(\omega_{0}+2 \pi j\right)
$$

then we have

$$
d_{j}^{2}=c_{j}^{2} \widehat{\varphi}_{1}\left(\omega_{0}+2 \pi j\right)+c_{j}^{3} \widehat{\varphi}_{2}\left(\omega_{0}+2 \pi j\right)
$$

$$
S\left(\omega_{0}\right)=\sum_{j \in \mathbb{Z}^{2}}\left\{d_{j}^{1} \widehat{\varphi}_{1}\left(\omega_{0}+2 \pi j\right)+d_{j}^{2} \widehat{\varphi}_{2}\left(\omega_{0}+2 \pi j\right)\right\}=0 .
$$

Now we show that at least $d_{j_{0}}^{1} \neq 0$ or $d_{j_{0}}^{2} \neq 0$, for this consider the case $r_{0}=1$, i.e., $c_{j_{0}}^{1} \neq 0$.

If we suppose that $d_{j_{0}}^{1}=c_{j_{0}}^{1} \widehat{\varphi}_{1}\left(\omega_{0}+2 \pi j_{0}\right)+c_{j_{0}}^{2} \widehat{\varphi}_{2}\left(\omega_{0}+2 \pi j_{0}\right)=0$, then

$$
\widetilde{S}\left(\omega_{0}\right)=\sum_{j \in \mathbb{Z}^{2}}\left\{\widetilde{d}_{j}^{1} \widehat{\varphi}_{1}\left(\omega_{0}+2 \pi j\right)+\widetilde{d}_{j}^{2} \widehat{\varphi}_{2}\left(\omega_{0}+2 \pi j\right)\right\}=0
$$

where $\widetilde{d}_{j}^{1}=\widetilde{d}_{j}^{2}=0$ for all $j \in \mathbb{Z}^{2} \backslash\left\{j_{0}\right\}$ and $\widetilde{d}_{j_{0}}^{1}=c_{j_{0}}^{1} \neq 0, \widetilde{d}_{j_{0}}^{2}=c_{j_{0}}^{2}$.

Consequently there exist $\omega_{0} \in \mathbb{R}^{2}$ and $j_{0} \in \mathbb{Z}^{2}$ such that $\widetilde{S}\left(\omega_{0}\right)=0$ with $\widetilde{d}_{j_{0}}^{1} \neq 0$, which is absurd because the linear piecewise function vector $\Phi$ is stable, i.e., the sequences

$$
\left(\widehat{\varphi}_{r}(\omega+2 \pi j)\right)_{j \in \mathbb{Z}^{2}} \quad r=1,2
$$


are linearly independent for every $\omega \in \mathbb{R}^{2}$. Therefore $d_{j_{0}}^{1} \neq 0$. A similar technique can be applied in the cases of $r_{0}=2$ or 3 . So that, there exist $j_{0}$ such that at least $d_{j_{0}}^{1} \neq 0$ or $d_{j_{0}}^{2} \neq 0$.

Consequently there exist $\omega_{0} \in \mathbb{R}^{2}, j_{0} \in \mathbb{Z}^{2}$ and $t_{0} \in\{1,2\}$ such that the equation (29) hold with $d_{j_{0}}^{t_{0}} \neq 0$, which is in contradiction with the fact that $\Phi$ is stable.

Theorem 2.6. The subdivision scheme associated to the quartic function vector $\Psi$ converges for all $\lambda \in$ $\left(\ell^{\infty}\left(\mathbb{Z}^{2}\right)\right)^{3}$, and the limit function is $\mathcal{C}^{2}$ quartic spline in the four-directional mesh given by

$$
S_{\lambda}=\sum_{j \in \mathbb{Z}^{2}} \lambda_{j}^{1} \psi_{1}(\cdot-j)+\sum_{j \in \mathbb{Z}^{2}} \lambda_{j}^{2} \psi_{2}(\cdot-j)+\sum_{j \in \mathbb{Z}^{2}} \lambda_{j}^{3} \psi_{3}(\cdot-j) .
$$

Proof. Let $Q_{i}=\sum_{j \in \mathbb{Z}^{2}} P_{i-2 j}$, for all $i \in \mathbb{Z}^{2}$ there exist $\mu \in \mathbb{Z}^{2}$ and $\nu \in\{(0,0),(1,0),(0,1),(1,1)\}$ such that $i=2 \mu+\nu$, so that we have $Q_{i}=Q_{\nu}$. Then, it suffices to prove that

$$
\mathbf{v} Q_{\nu}=\mathbf{v}, \quad \text { for } \mathbf{v}=(1,1,1), \text { and } \nu \in\{(0,0),(1,0),(0,1),(1,1)\} .
$$

By using the expression of $P_{i-2 j}$ given in Theorem 2.3 we have

$$
Q_{(0,0)}=\left[\begin{array}{ccc}
\frac{1}{4} & \frac{1}{8} & \frac{1}{4} \\
\frac{1}{2} & \frac{1}{2} & 0 \\
\frac{1}{4} & \frac{3}{8} & \frac{3}{4}
\end{array}\right], Q_{(1,0)}=Q_{(0,1)}=Q_{(1,1)}=\left[\begin{array}{ccc}
\frac{1}{4} & \frac{1}{8} & 0 \\
\frac{1}{2} & \frac{1}{2} & \frac{1}{2} \\
\frac{1}{4} & \frac{3}{8} & \frac{1}{2}
\end{array}\right] .
$$

and we can easily verify that the matrices $Q_{\nu}$, for $\nu \in\{(0,0),(1,0),(0,1),(1,1)\}$, have $\mathbf{v}$ as a left eigenvector associated with the eigenvalue 1 , and the conditions (27) are satisfied. Since $\Psi$ is stable, we obtain, from the lemma, 2.4 the enounced result.

We apply the subdivision scheme (26) for the initial sequences $\lambda_{j}^{(0)}=\left(\delta_{j}, 0,0\right)^{T}, \lambda_{j}^{(0)}=\left(0, \delta_{j}, 0\right)^{T}$ and $\lambda_{j}^{(0)}=\left(0,0, \delta_{j}\right)^{T}$ to compute the B-splines $\psi_{1}, \psi_{2}$ and $\psi_{3}$ respectively. In figures 5,6 and 7 , we give the graph of these B-splines approximated by the iterates sequences $\lambda_{j}^{(8)}$ in the three cases.

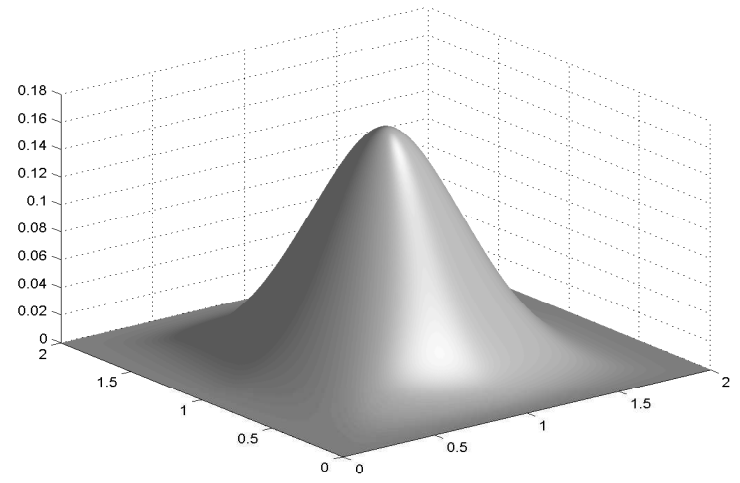

FiguRE 5. Graph of the B-spline $\psi_{1}$.

Remark 2.7. In order to construct the initial function spline $S_{\lambda}=\sum_{j \in \mathbb{Z}^{2}} \lambda_{j}^{T} \Psi(\cdot-j)$ in the subdivision scheme, we develop in the further work the construction of spline quasi-interpolants with optimal approximation orders and 


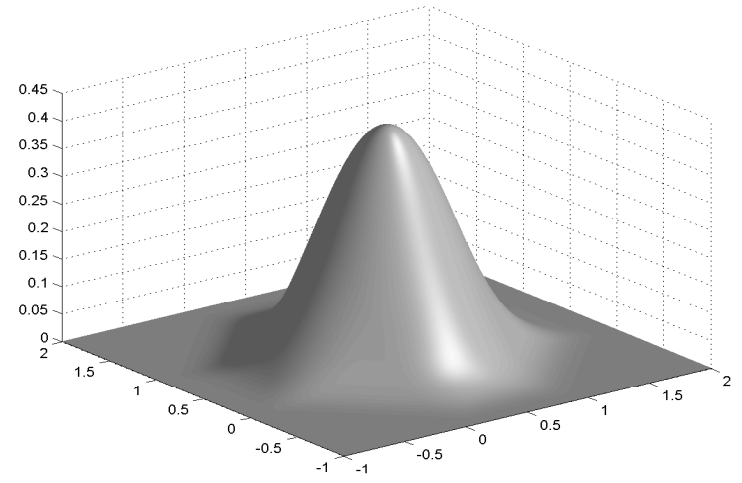

Figure 6. Graph of the B-spline $\psi_{2}$.

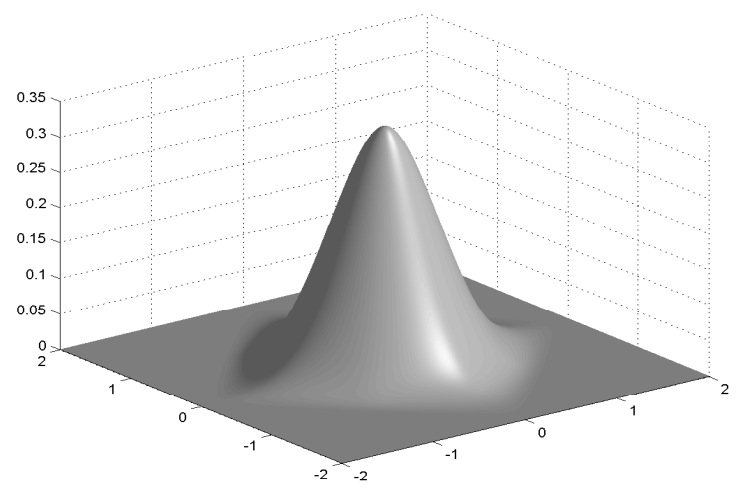

Figure 7. Graph of the B-spline $\psi_{3}$.

small norms in the space $\mathcal{S}(\Psi)$. These operators, so-called near-best quasi-interpolants, are useful in several applications like approximation and estimation, numerical quadrature and numerical solutions of integral or partial differential equations.

Remark 2.8. The results described above can be extended to various cases in the spline space $\mathcal{S}_{4 k+4}^{3 k+2}(\tau)$ of class $\mathcal{C}^{3 k+2}$ and degree $4 k+4, k \in \mathbf{N}$. In this space there are three independent locally supported B-splines, two are minimally supported, $\psi_{1}^{k}$ and $\psi_{2}^{k}$, and one is quasi-minimally supported $\psi_{3}^{k}$ defined by:

$$
\psi_{i}^{k}:= \begin{cases}\psi_{i}, & \text { for } k=0, \\ \psi_{i} * B_{k, k, k, k}, & \text { for } k=1,2, \ldots\end{cases}
$$

for $i=1,2,3$, where $B_{k, l, m, n}:=B_{X_{k, l, m, n}}, k, l, m, n \in \mathbb{N}$, is the bivariate box-spline on the four-directional mesh $\tau$, associated with the directional set

$$
X_{k, l, m, n}=\{\underbrace{e_{1}, \ldots, e_{1}}_{k}, \underbrace{e_{2}, \ldots, e_{2}}_{l}, \underbrace{e_{3}, \ldots, e_{3}}_{m}, \underbrace{e_{4}, \ldots, e_{4}}_{n}\}
$$


defined as the inverse Fourier transform of

$$
\begin{aligned}
\widehat{B}_{k, l, m, n}(w) & =\widehat{B}_{X_{k, l, m, n}}(w):=\int_{\mathbb{R}^{2}} B_{X_{k, l, m, n}}(x) e^{-i w \cdot x} d x \\
& =\left(\frac{1-e^{-i w_{1}}}{i w_{1}}\right)^{k}\left(\frac{1-e^{-i w_{2}}}{i w_{2}}\right)^{l}\left(\frac{1-e^{-i\left(w_{1}+w_{2}\right)}}{i\left(w_{1}+w_{2}\right)}\right)^{m}\left(\frac{1-e^{-i\left(w_{1}-w_{2}\right)}}{i\left(w_{1}-w_{2}\right)}\right)^{n}
\end{aligned}
$$

with $w=\left(w_{1}, w_{2}\right)$.

If we put $\Psi^{k}=\left(\psi_{1}^{k}, \psi_{2}^{k}, \psi_{3}^{k}\right)^{T}$, then $\Psi^{k}=\Psi * B_{k, k, k, k}$. So that, by using Theorem 2.3, Lemma 2.1 and the fact that the box-spline $B_{k, k, k, k}$ is a refinable function, we can prove that the spline function vector $\Psi^{k}$ is a refinable function and we can determine its refinement matrix mask by using the equation (21). But in this case the support of the mask is larger.

Remark 2.9. If we define

$$
\mathcal{S}^{k}:=\mathcal{S}^{k}(\Psi):=\left\{f\left(2^{k} \cdot\right): f \in \mathcal{S}(\Psi)\right\}
$$

then

$$
\mathcal{S}^{k} \subset \mathcal{S}^{k+1}
$$

and since $\Psi$ is compactly supported function vector, we deduce from [17] that

$$
\overline{\bigcup_{k \in \mathbb{Z}} \mathcal{S}^{k}}=L_{2}\left(\mathbb{R}^{2}\right) \text { and } \bigcap_{k \in \mathbb{Z}} \mathcal{S}^{k}=\{0\} \text {. }
$$

Furthermore, since the refinable function vector $\Psi$ is stable, the sequences of subspaces $\left(\mathcal{S}^{k}\right)_{k}$ form a multiresolution of $L_{2}\left(\mathbb{R}^{2}\right)$. The construction of the associated compactly supported multiwavelets and prewavelets will be studied in a future work.

\section{"Research supported in part by PROTARS III, D11/18"}

\section{REFERENCES}

[1] D. Barrera, M.J. Ibáñez, P. Sablonnière, Near-best quasi-interpolants on uniform and nonuniform partitions, in: A. Cohen, J.L. Merrien, L.L. Schumaker (Eds), Curve and Surface Fitting, Saint-Malo 2002, Nashboro Press, Brentwood, pp. 31-40, 2003.

[2] D. Barrera, M.J. Ibáñez, P. Sablonnière, D. Sbibih, Near minimally normed discrete and integral spline quasi-interpolants on uniform partitions of real line, J. Comput. Appl. Math., 2004.

[3] D. Barrera, M.J. Ibáñez, P. Sablonnière, D. Sbibih, Near-best quasi-interpolants associated with H-splines on a three directional mesh, J. Comput. Appl. Math., 2005.

[4] A. S. Cavaretta, W. Dahmen, and C.A. Micchelli, Stationary subdivision, Mem. Amer. Math. Soc., American Math. Soc, Providence, 93, 1991.

[5] D. R. Chen, R. Q. Jia, and S. D. Riemenschneider, Convergence of vector subdivision schemes in Sobolev spaces, Appl. Comput. Harmon. Anal., 12, 128-149, 2002.

[6] C. K. Chui, Multivariate splines, CBMS-NSF Regional Conference Series in Applied Mathematics, SIAM, Philadelphia, 1988.

[7] C. K. Chui and T. X. He, On minimal and quasi-minimal supported bivariate splines, J. Approx. Theory, 52, pp. 217-238, 1988.

[8] C. K. Chui, M.-J. Lai, Algorithms for generating B-nets and graphically displaying spline surfaces on three- and four-directional meshes, Comput. Aided Geom. Design 8, pp. 479-493, 1991.

[9] C. Conti and K. Jetter, A New subdivision method for bivariate splines on the four-directional mesh, J. Comput. Appl. Math., n. 119, pp. 81-96, 2000.

[10] C. Conti, K. Jetter, A Note on Convolving Refinable Function Vectors, In Curve and Surfaces Fitting, A. Coehn, C. Rabut, L.L.Schumaker eds. pp. 135-142, 2000.

[11] N. Dyn, D. Levin, Subdivision schemes in geometric modelling, In Acta Numerica, A. Iserles (ed.), Cambridge University Press (2002), 73-144.

[12] T. N. T. Goodman and S. L. Lee, Convergence of cascade algorithms, In Mathematical methods for curves and surfaces II, Innov. Appl. Math., Vanderbilt Univ. Press, Nashville, pp. 191-212, 1998. 
[13] B. Han, Vector Cascade Algorithms and Refinable Function Vectors in Sobolev Spaces, J. Approx. Theory, 124, 1, 44-88, 2003.

[14] K. Höllig, Box splines, in Approximation Theory V, C. K. Chui, L. L. Schumaker, J. D. Ward (eds.), Academic Press, New York, pp. 71-95, 1986.

[15] R.A. Horn, C.R. Johnson, Topics in matrix analysis. Cambridge University Press, 1991.

[16] R. Q. Jia and C. A. Micchelli, Using the refinement equations for the construction of pre-wavelets II: powers of two, in Curves and Surfaces (P.-J. Laurent, A. Le Méhauté, and L. L. Schumaker, eds), Academic Press (New York), 209-246, 1991.

[17] R. Q. Jia and Z. Shen, Multiresolution and wavelets, Proc. Edinburgh Math. Soc., 37, 271-300, 1994.

[18] P. Sablonnière, Bases de Bernstein et approximants splines. Th'ese de Doctorat, Universit e de Lille, 1982.

[19] P. Sablonnière, Bernstein-Bézier methods for the construction of bivariate spline approximants, CAGD 2, pp. 29-36, 1985.

[20] P. Sablonnière, Quasi-interpolants associated to H-splines on a three direction mesh, J. Comput. Appl. Math. 66, pp.433-442, 1996.

[21] P. Sablonnière, H-splines and quasi-interpolants on a three directional mesh, in Advanced Problems in Constructive Approximation, M. D. Buhmann and D. H. Mache (eds.), International Series of Numerical Mathematics Vol. 142, Birkhäuser Verlag, Basel., pp. 187-201, 2002.

[22] Z. W. Shen, Refinable function vectors, SIAM J. Math. Anal., 29, 235-250, 1998.

[23] Ch. Van Loan, Computational frameworks for the fast Fourier transform. SIAM, Philadelphia, 1992. 